\title{
A Generalization of the Cayley-Hamilton Theorem
}

\author{
Raj Kumar Kanwar \\ Department of Mathematics and Statistics, Himachal Pradesh University, Shimla, India \\ Email: rjkmrkanwar@yahoo.co.in
}

Received June 21, 2012; revised August 26, 2012; accepted September 11, 2012

\begin{abstract}
It is proposed to generalize the concept of the famous classical Cayley-Hamilton theorem for square matrices wherein for any square matrix $A$, the det $(A-x I)$ is replaced by det $f(x)$ for arbitrary polynomial matrix $f(x)$.
\end{abstract}

Keywords: Polynomial Matrix; Square Matrix; Non-Singular Matrix; Adjoint of a Matrix; Leading Coefficient Matrix

\section{Introduction}

The classical Cayley-Hamilton theorem [1-4] says that every square matrix satisfies its own characteristic equation. The Cayley-Hamilton theorem has been extended to rectangular matrices $[5,6]$, block matrices $[7,8]$, pairs of commuting matrices [9-11] and standard and singular two-dimensional linear systems [5,12]. The CayleyHamilton theorem has been extended to n-dimensional systems [13]. An extension of the Cayley-Hamilton theorem for $2 \mathrm{D}$ continuous discrete-time linear systems has been given in [14].

The Cayley-Hamilton theorem and its generalizations have been used in control systems $[14,15]$ and also automation and control in $[16,17]$, electronics and circuit theory [6], time-systems with delays [18-20], singular 2-D linear systems [5], 2-D continuous discrete linear systems [12], automation and electrotechnics [21], etc.

In this paper an overview of generalization of the Cayley-Hamilton theorem is presented. The linear polynomial matrix $(A-x I)$ of det $(A-x I)$ in the classical Cayley-Hamilton theorem is replaced by the general polynomial matrix

$$
f(x)=A_{0}+A_{1} x+\cdots+A_{n} x^{n},
$$

where $A_{i}^{\prime} s$ for $i=0,1,2, \cdots, n$ are square matrices of the same order. In the Theorem 1 given below it is proved that if $f(x)=\operatorname{det} f(x)$ and whenever for a square matrix $A f(A)=O$ implies $g(A)=O$ also. The converse of Theorem 1 is not true, is illustrated with the help of examples 1 and 2 in which the leading coefficient matrix of the polynomial matrix $f(x)$ may be singular or non-singular. A relation between the coefficients of the polynomial $g(x)$ and the coefficient matrices of $f(x)$ is worked out in corollaries 1,2 and 3 .

\section{Preliminaries}

Lemma 1. If the elements of a matrix $A$ are polynomials in $x$ of degree $\leq n$, then $A$ can be expressed as a polynomial matrix $A_{0}+A_{1} x+A_{2} x^{2}+\cdots+A_{n} x^{n}$ in $x$ of degree $\leq$ $n$, where the matrices $A_{i}^{\prime} s$ are of the same order as that of the matrix $A$.

Illustration 1. Let

$$
A=\left(\begin{array}{ccc}
x+2 x^{3} & -5 & -3+2 x \\
-5 x & x-2 x^{2} & 3+4 x^{3} \\
2-3 x+4 x^{2} & 4-2 x & x^{2}-x^{3}
\end{array}\right)
$$

be a matrix of order $3 \times 3$. Then

$$
A=A_{0}+A_{1} x+A_{2} x^{2}+A_{3} x^{3},
$$

where

$$
\begin{aligned}
& A_{0}=\left(\begin{array}{ccc}
0 & -5 & -3 \\
0 & 0 & 3 \\
2 & 4 & 0
\end{array}\right) ; A_{1}=\left(\begin{array}{ccc}
1 & 0 & 2 \\
-5 & 1 & 0 \\
-3 & -2 & 0
\end{array}\right) ; \\
& A_{2}=\left(\begin{array}{ccc}
0 & 0 & 0 \\
0 & -2 & 0 \\
4 & 0 & 1
\end{array}\right) \text { and } A_{3}=\left(\begin{array}{ccc}
2 & 0 & 0 \\
0 & 0 & 4 \\
0 & 0 & -1
\end{array}\right)
\end{aligned}
$$

Lemma 2. If $A$ is a square matrix of order $n$ having elements as polynomials in $x$ each of degree $\leq m$, then the elements of the adjoint of the matrix $A$ are also polynomials in $x$ of degree $\leq m(n-1)$.

Illustration 2. Let

$$
A=\left(\begin{array}{ccc}
x+2 x^{3} & -5 x^{4} & -3+2 x \\
-5 x & x-2 x^{2} & 3+4 x^{3} \\
2-3 x+4 x^{2} & 4-2 x & x^{4}-x^{3}
\end{array}\right)
$$


be a matrix of order $3 \times 3$ having elements as polynomials in $x$ of degree $\leq 4$, then

$$
\operatorname{adj} A=\left(\begin{array}{lll}
f_{11}\left(x^{6}\right) & f_{12}\left(x^{8}\right) & f_{13}\left(x^{7}\right) \\
f_{21}\left(x^{5}\right) & f_{22}\left(x^{7}\right) & f_{23}\left(x^{6}\right) \\
f_{31}\left(x^{4}\right) & f_{32}\left(x^{6}\right) & f_{33}\left(x^{5}\right)
\end{array}\right),
$$

where $f_{i, j}\left(x^{r}\right)$ denotes the $(i, j)$ th element of the $\operatorname{adj} A$, a polynomial in $x$ of degree $\leq r$. For instance in $\operatorname{adj} A$, the element at the (2.1) th position is

$$
f_{21}\left(x^{5}\right)=6-9 x+12 x^{2}+8 x^{3}-17 x^{4}+21 x^{5} .
$$

Hence by the Lemma 1 , because adjA contains elements as polynomials in $x$ of degree $\leq 8$, it implies that $\operatorname{adj}(A)=B_{0}+B_{1} x+B_{2} x^{2}+\cdots+B_{8} x^{8}$, where each of the $B_{i}^{\prime} s,(0 \leq i \leq 8)$ is also a square matrix of order 3 .

Remark 1. Prior to understand the concept in the proof of the main Theorem 1 given below, we first consider the following two illustrations of polynomial matrix $f(x)$ having the leading coefficient matrix singular or non-singular such that if $g(x)=\operatorname{det} f(x)$ and for a square matrix $A$, whenever

$$
f(A)=O \Rightarrow g(A)=O .
$$

Illustration 3: Let

$$
f(x)=A_{0}+A_{1} x+A_{2} x^{2}
$$

be a polynomial matrix over $M_{2}(F[x])$ for

$$
A_{0}=\left(\begin{array}{ll}
3 & 3 \\
4 & 1
\end{array}\right), A_{1}=\left(\begin{array}{ll}
1 & -2 \\
3 & -1
\end{array}\right) \text { and } A=\left(\begin{array}{ll}
-2 & 1 \\
-1 & 0
\end{array}\right),
$$

where $A_{2}$ is a non-singular matrix and $M_{2}(F[x])$ denotes the set of all $2 \times 2$ matrices whose elements are polynomials in $x$ over the field $F$. Then there exists a matrix $A=\left(\begin{array}{cc}-1 & 2 \\ 0 & 3\end{array}\right)$ such that;

$$
\begin{aligned}
f(A) & =A_{0}+A_{1} A+A_{2} A^{2} \\
& =\left(\begin{array}{ll}
3 & 3 \\
4 & 1
\end{array}\right)+\left(\begin{array}{cc}
1 & -2 \\
3 & -1
\end{array}\right)\left(\begin{array}{cc}
-1 & 2 \\
0 & 3
\end{array}\right)+\left(\begin{array}{ll}
-2 & 1 \\
-1 & 0
\end{array}\right)\left(\begin{array}{ll}
1 & 4 \\
0 & 9
\end{array}\right) \\
& =\left(\begin{array}{ll}
3 & 3 \\
4 & 1
\end{array}\right)+\left(\begin{array}{cc}
-1 & -4 \\
-3 & 3
\end{array}\right)+\left(\begin{array}{cc}
-2 & 1 \\
-1 & -4
\end{array}\right)=O
\end{aligned}
$$

Also from (2.1), we have

$$
\begin{aligned}
f(x)=\left(\begin{array}{ll}
3 & 3 \\
4 & 1
\end{array}\right)+\left(\begin{array}{ll}
1 & -2 \\
3 & -1
\end{array}\right) x+\left(\begin{array}{ll}
-2 & 1 \\
-1 & 0
\end{array}\right) x^{2}=\left(\begin{array}{cc}
3+x-2 x^{2} & 3-2 x+x^{2} \\
4+3 x-x^{2} & 1-x
\end{array}\right) \\
\Rightarrow g(x)=\operatorname{det} f(x)=\left(3+x-2 x^{2}\right) \cdot(1-x)-\left(4+3 x-x^{2}\right) \cdot\left(3-2 x+x^{2}\right)=-9-3 x+2 x^{2}-3 x^{3}+x^{4} . \\
\Rightarrow g(A)=-9 I-3 A+2 A^{2}-3 A^{3}+A^{4}=-9\left(\begin{array}{ll}
1 & 0 \\
0 & 1
\end{array}\right)-3\left(\begin{array}{cc}
-1 & 2 \\
0 & 3
\end{array}\right)+2\left(\begin{array}{ll}
1 & 4 \\
0 & 9
\end{array}\right)-3\left(\begin{array}{cc}
-1 & 14 \\
0 & 27
\end{array}\right)+\left(\begin{array}{ll}
1 & 40 \\
0 & 81
\end{array}\right) \\
=\left(\begin{array}{cc}
-9 & 0 \\
0 & -9
\end{array}\right)+\left(\begin{array}{ll}
3 & -6 \\
0 & -9
\end{array}\right)+\left(\begin{array}{cc}
2 & 8 \\
0 & 18
\end{array}\right)+\left(\begin{array}{cc}
3 & -42 \\
0 & -81
\end{array}\right)+\left(\begin{array}{ll}
1 & 40 \\
0 & 81
\end{array}\right)=O .
\end{aligned}
$$

Hence, $f(A)=O$ implies $g(A)=O$.

Illustration 4: Consider the polynomial matrix

$$
f(x)=A_{0}+A_{1} x+A_{2} x^{2}
$$

over $M_{2}(F[x])$, for $A_{0}=\left(\begin{array}{cc}150 & -97 \\ 86 & -55\end{array}\right) ; \quad A_{1}=\left(\begin{array}{cc}1 & 2 \\ -1 & 4\end{array}\right)$ and $A_{2}=\left(\begin{array}{ll}3 & 9 \\ 2 & 6\end{array}\right)$, where the leading coefficient matrix $A_{2}$ is singular. Then there exists a matrix $A=\left(\begin{array}{cc}-2 & 1 \\ 4 & -3\end{array}\right)$ such that

$$
\begin{aligned}
f(A) & =A_{0}+A_{1} A+A_{2} A^{2}=\left(\begin{array}{cc}
150 & -97 \\
86 & -55
\end{array}\right)+\left(\begin{array}{cc}
1 & 2 \\
-1 & 4
\end{array}\right)\left(\begin{array}{cc}
-2 & 1 \\
4 & -3
\end{array}\right)+\left(\begin{array}{cc}
3 & 9 \\
2 & 6
\end{array}\right)\left(\begin{array}{cc}
8 & -5 \\
-20 & 13
\end{array}\right) \\
& =\left(\begin{array}{cc}
150 & -97 \\
86 & -55
\end{array}\right)+\left(\begin{array}{cc}
6 & -5 \\
18 & -13
\end{array}\right)+\left(\begin{array}{cc}
-156 & 102 \\
-104 & 68
\end{array}\right)=O .
\end{aligned}
$$

From (2.2), we have

$$
\begin{aligned}
f(x) & =\left(\begin{array}{cc}
150 & -97 \\
86 & -55
\end{array}\right)+\left(\begin{array}{cc}
1 & 2 \\
-1 & 4
\end{array}\right) x+\left(\begin{array}{ll}
3 & 9 \\
2 & 6
\end{array}\right) x^{2}=\left(\begin{array}{cc}
150+x+3 x^{2} & -97+2 x+9 x^{2} \\
86-x+2 x^{2} & -55+4 x+6 x^{2}
\end{array}\right) \Rightarrow g(x)=\operatorname{det} f(x) \\
& =\left(150+x+3 x^{2}\right)\left(-55+4 x+6 x^{2}\right)-\left(86-x+2 x^{2}\right)\left(-97+2 x+9 x^{2}\right)=92+276 x+161 x^{2}+23 x^{3} .
\end{aligned}
$$


As in Illustration 3 , it can be easily verified that

$$
g(A)=92 I+276 A+161 A^{2}+23 A^{3}=O .
$$

\section{Main Results}

Theorem 1. Let $f(x)=A_{0}+A_{1} x+A_{2} x^{2}+\cdots+A_{m} x^{m}$ be a polynomial matrix for $f(x) \in M_{n}(F[x])$ where $A_{i}^{\prime} s \in M_{n}(F)$ for $i=1,2,3, \cdots, m$, are square matrices of order $n$ over the field $F$. If $g(x)=\operatorname{det} f(x)$, then whenever $f(A)=O$ (Zero matrix) implies $g(A)=O$. Converse is not true.

\section{Proof. Since}

$$
f(x)=A_{0}+A_{1} x+A_{2} x^{2}+\cdots+A_{m} x^{m}
$$

is itself is a matrix of order $n \times n$ having elements as polynomials in $x$ each of degree $\leq m$, therefore, using lemma 2, we have

$$
\operatorname{adj} f(x)=B_{0}+B_{1} x+B_{2} x^{2}+\cdots+B_{m(n-1)} x^{m(n-1)}
$$

Also $g(x)=\operatorname{det} f(x)$ is a polynomial in $x$ over $F[x]$ of degree $\leq m n$. Therefore, using Lemma 1 , we have

$$
g(x)=\operatorname{det} f(x)=p_{0}+p_{1} x+p_{2} x^{2}+p_{3} x^{3}+\cdots+p_{m n} x^{m n}
$$

Since for any square matrix $A$, we have;

$$
A(\operatorname{adj} A)=(\operatorname{adj} A) A=|A| I
$$

where $I$ is the identity matrix of the same order as of $A$. Now using (3.4), we have

$$
f(x) \operatorname{adj} f(x)=g(x) I
$$

Therefore, using (3.1) to (3.3) above, we have from (3.5)

$$
\begin{aligned}
& \left(A_{0}+A_{1} x+A_{2} x^{2}+\cdots+A_{m} x^{m}\right) \\
& \cdot\left(B_{0}+B_{1} x+B_{2} x^{2}+\cdots+B_{m(n-1)} x^{m(n-1)}\right) \\
& =\left(p_{0}+p_{1} x+p_{2} x^{2}+p_{3} x^{3}+\cdots+p_{m n} x^{m n}\right) I .
\end{aligned}
$$

Comparing coefficients of the corresponding terms on both sides of Equation (3.6), we get

$$
\left.\begin{array}{l}
A_{0} B_{0}=p_{0} I \\
A_{0} B_{1}+A_{1} B_{0}=p_{1} I \\
A_{0} B_{2}+A_{1} B_{1}+A_{2} B_{0}=p_{2} I \\
A_{0} B_{3}+A_{1} B_{2}+A_{2} B_{1}+A_{3} B_{0}=p_{3} I \\
\vdots \\
A_{0} B_{m}+A_{1} B_{m-1}+A_{2} B_{m-2}+\cdots+A_{m} B_{0}=p_{m} I \\
A_{0} B_{m+1}+A_{1} B_{m}+A_{2} B_{m-1}+\cdots+A_{m} B_{1}=p_{m+1} I \\
\vdots \\
A_{m-2} B_{m n-m}+A_{m-1} B_{m n-m-1}+A_{m} B_{m n-m-2}=p_{m n-2} I \\
A_{m-1} B_{m n-m}+A_{m} B_{m n-m-1}=p_{m n-1} I \\
A_{m} B_{m n-m}=p_{m n} I
\end{array}\right\}
$$

Multiplying the equations in (3.7) by the matrices

$$
I, A, A^{2}, A^{3}, \cdots, A^{m}, A^{m+1}, \cdots, A^{m n-2}, A^{m n-1}, A^{m n}
$$

respectively and adding, we obtain;

$$
\begin{aligned}
& f(A)\left\{B_{0}+A B_{1}+A^{2} B_{2}+A^{3} B_{3}+\cdots\right. \\
& \left.+A^{m n-m-1} B_{m n-m-1}+A^{m n-m} B_{m n-m}\right\} \\
& =p_{0} I+p_{1} A+p_{2} A^{2}+p_{3} A^{3}+\cdots+p_{m n} A^{m n}=g(A) \\
& \Rightarrow g(A)=f(A)\left\{B_{0}+A B_{1}+A^{2} B_{2}+A^{3} B_{3}+\cdots\right. \\
& \left.+A^{m n-m-1} B_{m n-m-1}+A^{m n-m} B_{m n-m}\right\}=O
\end{aligned}
$$

Converse is not true. For this consider the following examples with the coefficient matrix singular and nonsingular respectively.

Example 1. Consider the function $f(x)=A_{0}+A_{1} x$; where

$$
\begin{aligned}
A_{0}=\left(\begin{array}{cc}
2 & -3 \\
4 & 7
\end{array}\right) ; A_{1}=\left(\begin{array}{cc}
-3 & 12 \\
2 & -8
\end{array}\right)(\text { singular }) \\
\begin{aligned}
\Rightarrow f(x) & =\left(\begin{array}{cc}
2 & -3 \\
4 & 7
\end{array}\right)+\left(\begin{array}{cc}
-3 & 12 \\
2 & -8
\end{array}\right) x \\
& =\left(\begin{array}{cc}
2-3 x & -3+12 x \\
4+2 x & 7-8 x
\end{array}\right) \\
\Rightarrow g(x) & =\operatorname{det} f(x) \\
& =(2-3 x)(7-8 x)-(4+2 x)(-3+12 x) \\
& =26-79 x .
\end{aligned}
\end{aligned}
$$

Then for the scalar matrix $A=\frac{26}{79} I_{2}$, we have $g(A)=26 I-26 I=O$. Whereas,

$$
\begin{aligned}
f(A) & =\left(\begin{array}{cc}
2 & -3 \\
4 & 7
\end{array}\right)+\left(\begin{array}{cc}
-3 & 12 \\
2 & -8
\end{array}\right) \frac{26}{79} I \\
& =\left(\begin{array}{cc}
2 & -3 \\
4 & 7
\end{array}\right)+\frac{26}{79}\left(\begin{array}{cc}
-3 & 12 \\
2 & -8
\end{array}\right)=\frac{1}{79}\left(\begin{array}{cc}
80 & 75 \\
368 & 345
\end{array}\right) \neq O .
\end{aligned}
$$

Example 2: Consider the function $f(x)=A_{0}+A_{1} x+A_{2} x^{2}$; where

$$
\begin{aligned}
& A_{0}=\left(\begin{array}{ll}
1 & 0 \\
0 & 6
\end{array}\right) ; A_{1}=\left(\begin{array}{ll}
0 & 0 \\
0 & 0
\end{array}\right) \text { and } \\
& A_{2}=\left(\begin{array}{ll}
-1 & 2 \\
-2 & 5
\end{array}\right) \text { (non-singular) } \\
& \Rightarrow f(x)=\left(\begin{array}{cc}
1-x^{2} & 2 x^{2} \\
-2 x^{2} & 6+5 x^{2}
\end{array}\right) \\
& \Rightarrow g(x)=\operatorname{det} f(x)=\left(1-x^{2}\right)\left(6+5 x^{2}\right)+4 x^{4} \\
& \quad=6-x^{2}-x^{4}=-\left(x^{4}+x^{2}-6\right) .
\end{aligned}
$$


Then there exist infinite number of matrices $A$ over the complex numbers $C$ of the form

$$
A=\left\{\begin{array}{cc}
\left(\begin{array}{cc} 
\pm \sqrt{2-a b} & a \\
b & \mp \sqrt{2-a b}
\end{array}\right) ; & \text { if } a^{2}+b^{2} \neq 0 \\
\left(\begin{array}{cc} 
\pm \sqrt{2} & 0 \\
0 & \pm \sqrt{2}
\end{array}\right) ; & \text { if } a^{2}+b^{2}=0
\end{array}\right.
$$

or

$$
A=\left\{\begin{array}{cc}
\left(\begin{array}{cc} 
\pm \sqrt{-3-a b} & a \\
b & \mp \sqrt{-3-a b}
\end{array}\right) ; & \text { if } a^{2}+b^{2} \neq 0 \\
\left(\begin{array}{cc} 
\pm \sqrt{3} \mathrm{i} & 0 \\
0 & \pm \sqrt{3} \mathrm{i}
\end{array}\right) ; & \text { if } a^{2}+b^{2}=0
\end{array}\right.
$$

for $a, b \in C$, such that $g(A)=0$ but $f(A) \neq 0$.

For instance, if $a=5, b=2-3 i$, then

$$
\begin{aligned}
A & =\left(\begin{array}{cc}
\sqrt{-3-a b} & a \\
b & -\sqrt{-3-a b}
\end{array}\right) \\
& =\left(\begin{array}{cc}
\sqrt{-13+15 i} & 5 \\
2-3 \mathrm{i} & -\sqrt{-13+15 i}
\end{array}\right) \\
\Rightarrow A^{2} & =\left(\begin{array}{cc}
-3 & 0 \\
0 & -3
\end{array}\right) \text { and } A^{4}=\left(\begin{array}{ll}
9 & 0 \\
0 & 9
\end{array}\right) \\
\Rightarrow g(A)=-\left\{A^{4}+A^{2}-6 I\right\} & =-\left\{\left(\begin{array}{ll}
9 & 0 \\
0 & 9
\end{array}\right)+\left(\begin{array}{cc}
-3 & 0 \\
0 & -3
\end{array}\right)-6\left(\begin{array}{ll}
1 & 0 \\
0 & 1
\end{array}\right)\right\}=O .
\end{aligned}
$$

Whereas,

$$
\begin{aligned}
f(A) & =A_{0}+A_{2} A^{2}=\left(\begin{array}{ll}
1 & 0 \\
0 & 6
\end{array}\right)+\left(\begin{array}{ll}
-1 & 2 \\
-2 & 5
\end{array}\right)\left(\begin{array}{cc}
-3 & 0 \\
0 & -3
\end{array}\right) \\
& =\left(\begin{array}{ll}
4 & -6 \\
6 & -9
\end{array}\right) \neq O .
\end{aligned}
$$

Illustration 5. For $m=3$ in Theorem 1, let

$$
f(x)=A_{0}+A_{1} x+A_{2} x^{2}+A_{3} x^{3}
$$

be a polynomial matrix in $M_{3}(F[x])$,where $A_{i} \in M_{3}(F)$ such that $f(A)=0$ for some square matrix $A$ of order 3 .

$$
\Rightarrow f(A)=A_{0}+A_{1} A+A_{2} A^{2}+A_{3} A^{3}=0 .
$$

Since the elements of the matrix $f(x)$ are polynomials in $x$ of degree $\leq 3$

$$
\Rightarrow g(x)=\operatorname{det} f(x)
$$

is a polynomial in $x$ over the field $F$ of degree $\leq 9$.
Therefore, let

$$
g(x)=p_{0}+p_{1} x+p_{2} x^{2}+p_{3} x^{3}+\cdots+p_{9} x^{9}
$$

Also each element of the $\operatorname{adj} f(x)$ being a polynomial in $x$ of $\operatorname{deg} \leq 6$. So by Lemma (2), let

$$
\operatorname{adj} f(x)=B_{0}+B_{1} x+B_{2} x^{2}+\cdots+B_{6} x^{6}
$$

Now using (3.4), we have

$$
\begin{aligned}
& \left(A_{0}+A_{1} x+A_{2} x^{2}+A_{3} x^{3}\right)\left(B_{0}+B_{1} x+B_{2} x^{2}+\cdots+B_{6} x^{6}\right) \\
& =\left(p_{0}+p_{1} x+p_{2} x^{2}+p_{3} x^{3}+\cdots+p_{9} x^{9}\right) I .
\end{aligned}
$$

Comparing the coefficients of the equivalent powers of $x$ on both sides, we have

$$
\left.\begin{array}{l}
A_{0} B_{0}=p_{0} I \\
A_{0} B_{1}+A_{1} B_{0}=p_{1} I \\
A_{0} B_{2}+A_{1} B_{1}+A_{2} B_{0}=p_{2} I \\
A_{0} B_{3}+A_{1} B_{2}+A_{2} B_{1}+A_{3} B_{0}=p_{3} I \\
A_{0} B_{4}+A_{1} B_{3}+A_{2} B_{2}+A_{3} B_{1}=p_{4} I \\
A_{0} B_{5}+A_{1} B_{4}+A_{2} B_{3}+A_{3} B_{2}=p_{5} I \\
A_{0} B_{6}+A_{1} B_{5}+A_{2} B_{4}+A_{3} B_{3}=p_{6} I \\
A_{1} B_{6}+A_{2} B_{5}+A_{3} B_{4}=p_{7} I \\
A_{2} B_{6}+A_{3} B_{5}=p_{8} I \\
A_{3} B_{6}=p_{9} I
\end{array}\right\}
$$

Multiplying these equations by $I, A, A^{2}, A^{3}, \cdots, A^{9}$ respectively and adding, we get;

$$
\begin{aligned}
& f(A)\left\{B_{0}+A B_{1}+A^{2} B_{2}+A^{3} B_{3}+A^{4} B_{4}+A^{5} B_{5}+A^{6} B_{6}\right\} \\
& =p_{0} I+p_{1} A+p_{2} A^{2}+p_{3} A^{3}+\cdots+p_{9} A^{9}=g(A) \\
& \Rightarrow g(A)=O(\because f(A)=0) .
\end{aligned}
$$

Corollary 1. If $f(x)$ and $g(x)$ be the polynomials given in (3.1) and (3.3) respectively, then for

$$
x=0 \Rightarrow g(0)=\operatorname{det} f(0) \Rightarrow p_{0}=\left|A_{0}\right| \text {. }
$$

Therefore, the constant term $p_{0}$ of the polynomial $g(x)$ is the determinant of the constant term $A_{0}$ in the polynomial matrix $f(x)$.

Corollary 2. From (3.1) and (3.3), for $\operatorname{det} f(x)=g(x)$, we have

$$
\begin{aligned}
& \operatorname{det}\left(A_{0}+A_{1} x+A_{2} x^{2}+\cdots+A_{m} x^{m}\right) \\
& =p_{0}+p_{1} x+p_{2} x^{2}+p_{3} x^{3}+\cdots+p_{m n} x^{m n} .
\end{aligned}
$$

Therefore, in case for $x=\frac{1}{y}$, when $x \rightarrow \infty$ or $y \rightarrow 0$, then from (3.13), we have 


$$
\begin{aligned}
& \operatorname{det}\left\{A_{0}+A_{1}\left(\frac{1}{y}\right)+A_{2}\left(\frac{1}{y}\right)^{2}+\cdots+A_{m}\left(\frac{1}{y}\right)^{m}\right\}=p_{0}+p_{1} \frac{1}{y}+p_{2} \frac{1}{y^{2}}+\cdots+p_{m n} \frac{1}{y^{m n}} \\
& \Rightarrow \operatorname{det}\left\{\frac{1}{y^{m}}\left(A_{0} y^{m}+A_{1} y^{m-1}+A_{2} y^{m-2}+\cdots+A_{m-1} y+A_{m}\right)\right\}=\frac{1}{y^{m n}}\left(p_{0} y^{m n}+p_{1} y^{m n-1}+p_{2} y^{m n-2}+\cdots+p_{m n-1} y+p_{m n}\right) \\
& \Rightarrow\left(\frac{1}{y^{m}}\right)^{n} \operatorname{det}\left(A_{0} y^{m}+A_{1} y^{m-1}+A_{2} y^{m-2}+\cdots+A_{m-1} y+A_{m}\right)=\frac{1}{y^{m n}}\left(p_{0} y^{m n}+p_{1} y^{m n-1}+p_{2} y^{m n-2}+\cdots+p_{m n-1} y+p_{m n}\right) \\
& \Rightarrow \operatorname{det}\left(A_{0} y^{m}+A_{1} y^{m-1}+A_{2} y^{m-2}+\cdots+A_{m-1} y+A_{m}\right)=p_{0} y^{m n}+p_{1} y^{m n-1}+p_{2} y^{m n-2}+\cdots+p_{m n-1} y+p_{m n} .
\end{aligned}
$$

Therefore, if $y \rightarrow 0$, then from (3.14), we get $p_{m n}=\left|A_{m}\right|$. Hence if, $\left|A_{m}\right|=0 \Rightarrow p_{m n}=0$.

Thus $\operatorname{deg} g(x)<m n$ if the leading coefficient matrix $A_{m}$ in $f(x)$ is singular.

Corollary 3. If

$$
f(x)=A_{0}+A_{1} x+A_{2} x^{2}+A_{3} x^{3}+A_{4} x^{4}
$$

be a bi-quadratic polynomial matrix for

$$
\begin{aligned}
& A_{0}=\left(\begin{array}{ll}
a_{0} & b_{0} \\
c_{0} & d_{0}
\end{array}\right) ; A_{1}=\left(\begin{array}{ll}
a_{1} & b_{1} \\
c_{1} & d_{1}
\end{array}\right) ; \\
& A_{2}=\left(\begin{array}{ll}
a_{2} & b_{2} \\
c_{2} & d_{2}
\end{array}\right) ; A_{3}=\left(\begin{array}{ll}
a_{3} & b_{3} \\
c_{3} & d_{3}
\end{array}\right) ; \\
& A_{4}=\left(\begin{array}{ll}
a_{4} & b_{4} \\
c_{4} & d_{4}
\end{array}\right)
\end{aligned}
$$

and if

$$
\begin{aligned}
g(x) & =\operatorname{det} f(x) \\
& =p_{0}+p_{1} x+p_{2} x^{2}+p_{3} x^{3}+\cdots+p_{8} x^{8}
\end{aligned}
$$

Then we have,

$$
\begin{aligned}
& A_{0}=\left[\begin{array}{ccc}
-1 & 2 & 0 \\
3 & 1 & -1 \\
2 & 0 & 3
\end{array}\right], A_{1}=\left[\begin{array}{ccc}
1 & 0 & -1 \\
2 & -2 & 1 \\
0 & -1 & 2
\end{array}\right], A_{2}=\left[\begin{array}{ccc}
1 & -2 & 3 \\
-1 & 0 & 2 \\
2 & 3 & -3
\end{array}\right], A_{3}=\left[\begin{array}{ccc}
3 & -2 & 1 \\
-1 & 0 & 3 \\
2 & 3 & 1
\end{array}\right] \\
& \Rightarrow f(x)=\left[\begin{array}{ccc}
-1+x+x^{2}+3 x^{3} & 2-2 x^{2}-2 x^{3} & -x+3 x^{2}+x^{3} \\
3+2 x-x^{2}-x^{3} & 1-2 x & -1+x+2 x^{2}+3 x^{3} \\
2+x^{2}+2 x^{3} & -x+2 x^{2}+3 x^{3} & 3+2 x-3 x^{2}+x^{3}
\end{array}\right] \\
& \Rightarrow g(x)=\operatorname{det} f(x)=-25-10 x+39 x^{2}+56 x^{3}-7 x^{4}+2 x^{5}+53 x^{6}-54 x^{7}-83 x^{8}-44 x^{9} \\
& =p_{0}+p_{1} x+p_{2} x^{2}+p_{3} x^{3}+p_{4} x^{4}+p_{5} x^{5}+p_{6} x^{6}+p_{7} x^{7}+p_{8} x^{8}+p_{9} x^{9} .
\end{aligned}
$$

where $p_{n}$, the coefficient of $x^{n}$ is given by

$$
\left.p_{n}=\sum\left|\begin{array}{ccc}
a_{i} & b_{j} & c_{k} \\
l_{i} & m_{j} & n_{k} \\
x_{i} & y_{j} & z_{k}
\end{array}\right| \text {, for } n=0,1,2, \cdots, 9 ; 0 \leq i, j, k \leq 3 \text { and } i+j+k=n\right\} \text {. }
$$

It can be easily verified that 


$$
\begin{aligned}
p_{0} & =\left|\begin{array}{ccc}
a_{0} & b_{0} & c_{0} \\
l_{0} & m_{0} & n_{0} \\
x_{0} & y_{0} & z_{0}
\end{array}\right|=\left|\begin{array}{ccc}
-1 & 2 & 0 \\
3 & 1 & -1 \\
2 & 0 & 3
\end{array}\right|=-25 . \\
p_{1} & =\left|\begin{array}{ccc}
a_{0} & b_{0} & c_{1} \\
l_{0} & m_{0} & n_{1} \\
x_{0} & y_{0} & z_{1}
\end{array}\right|+\left|\begin{array}{ccc}
a_{0} & b_{1} & c_{0} \\
l_{0} & m_{1} & n_{0} \\
x_{0} & y_{1} & z_{0}
\end{array}\right|+\left|\begin{array}{ccc}
a_{1} & b_{0} & c_{0} \\
l_{1} & m_{0} & n_{0} \\
x_{1} & y_{0} & z_{0}
\end{array}\right| \\
& =\left|\begin{array}{ccc}
-1 & 2 & -1 \\
3 & 1 & 1 \\
2 & 0 & 2
\end{array}\right|+\left|\begin{array}{ccc}
-1 & 0 & 0 \\
3 & -2 & -1 \\
2 & -1 & 3
\end{array}\right|+\left|\begin{array}{ccc}
1 & 2 & 0 \\
2 & 1 & -1 \\
0 & 0 & 3
\end{array}\right|=-10 .
\end{aligned}
$$

and

$$
\begin{aligned}
p_{2}= & \left|\begin{array}{ccc}
a_{0} & b_{0} & c_{2} \\
l_{0} & m_{0} & n_{2} \\
x_{0} & y_{0} & z_{2}
\end{array}\right|+\left|\begin{array}{ccc}
a_{0} & b_{2} & c_{0} \\
l_{0} & m_{2} & n_{0} \\
x_{0} & y_{2} & z_{0}
\end{array}\right|+\left|\begin{array}{ccc}
a_{2} & b_{0} & c_{0} \\
l_{2} & m_{0} & n_{0} \\
x_{2} & y_{0} & z_{0}
\end{array}\right| \\
& +\left|\begin{array}{ccc}
a_{0} & b_{1} & c_{1} \\
l_{0} & m_{1} & n_{1} \\
x_{0} & y_{1} & z_{1}
\end{array}\right|+\left|\begin{array}{ccc}
a_{1} & b_{0} & c_{1} \\
l_{1} & m_{0} & n_{1} \\
x_{1} & y_{0} & z_{1}
\end{array}\right|+\left|\begin{array}{ccc}
a_{1} & b_{1} & c_{0} \\
l_{1} & m_{1} & n_{0} \\
x_{1} & y_{1} & z_{0}
\end{array}\right| \\
= & \left|\begin{array}{ccc}
-1 & 2 & 3 \\
3 & 1 & 2 \\
2 & 0 & -3
\end{array}\right|+\left|\begin{array}{ccc}
-1 & -2 & 0 \\
3 & 0 & -1 \\
2 & 2 & 3
\end{array}\right|+\left|\begin{array}{ccc}
1 & 2 & 0 \\
-1 & 1 & -1 \\
1 & 0 & 3
\end{array}\right| \\
& +\left|\begin{array}{ccc}
-1 & 0 & -1 \\
3 & -2 & 1 \\
2 & -1 & 2
\end{array}\right|+\left|\begin{array}{ccc}
1 & 2 & -1 \\
2 & 1 & 1 \\
0 & 0 & 2
\end{array}\right|+\left|\begin{array}{ccc}
1 & 0 & 0 \\
2 & -2 & -1 \\
0 & -1 & 3
\end{array}\right| \\
= & 23+20+7+2-6-7=39 .
\end{aligned}
$$

Similarly coefficients of the other powers of $x$, i.e., $x^{3}, x^{4}, \cdots, x^{9}$ can be found by using (3.15). For instance

$$
p_{9}=\left|\begin{array}{ccc}
a_{3} & b_{3} & c_{3} \\
l_{3} & m_{3} & n_{3} \\
x_{3} & y_{3} & z_{3}
\end{array}\right|=\left|\begin{array}{ccc}
3 & -2 & 1 \\
-1 & 0 & 3 \\
2 & 3 & 1
\end{array}\right|=-44=\left|A_{3}\right|,
$$

which verifies our assertion.

\section{Conclusion}

The concept of the Theorem 1 given above and the relation in (3.15) can be generalized to any polynomial matrix of arbitrary degree with coefficients as square matrices of any order.

\section{Acknowledgements}

The author wishes to thank Dr. P. L. Sharma, Associate Professor Department of Mathematics and Statistics of the H. P. University Shimla (H.P.) India for his help and guidance. He also expresses his gratitude to the Govt. of Himachal Pradesh Department of Higher Education for granting him study leave to complete the assigned project.

\section{REFERENCES}

[1] J. Gilbert and L. Gilbert, "Linear Algebra and Matrix Theory," Academic Press Inc., New York, 1995.

[2] K. Hoffman and R. Kunze, "Linear Algebra," Prentice Hall of India, New Delhi, 2010.

[3] P. Lancaster, "Theory of Matrices," Academic Press, New York, 1969.

[4] F. R. Gantmatcher, "Theory of Matrices, Vol. 2," Chelsea Publishing, New York, 1974.

[5] T. Kaczorek, "An Existence of the Cayley-Hamilton Theorem for Singular 2-D Linear Systems with Non-Square Matrices," Bulletin of the Polish Academy of Sciences. Technical Sciences, Vol. 43, No. 1, 1995, pp. 39-48.

[6] T. Kaczorek, "Generalization of the Cayley-Hamilton Theorem for Non-Square Matrices," International Conference of Fundamentals of Electronics and Circuit Theory XVIIISPETO, Gliwice, 1995, pp. 77-83.

[7] T. Kaczorek, "An Existence of the Caley-Hamilton Theorem for Non-Square Block Matrices," Bulletin of the Polish Academy of Sciences. Technical Sciences, Vol. 43, No. 1, 1995, pp. 49-56.

[8] T. Kaczorek, "An Extension of the Cayley-Hamilton Theorem for a Standard Pair of Block Matrices," Applied Mathematics and Computation Sciences, Vol. 8, No. 3, 1998, pp. 511-516.

[9] F. R. Chang and C. N. Chan, "The Generalized CayleyHamilton Theorem for Standard Pencils," Systems \& Control Letters, Vol. 18, No. 3, 1992, pp. 179-182. doi:10.1016/0167-6911(92)90003-B

[10] F. L. Lewis, "Cayley-Hamilton Theorem and Fadeev's Method for the Matrix Pencil [sE-A]," $22^{\text {nd }}$ IEEE Conference on Decision and Control, San Diego, 1982, pp. 1282-1288.

[11] F. L. Lewis, "Further Remarks on the Cayley-Hamilton Theorem and Fadeev's Method for the Matrix Pencil [sEA]," IEEE Transactions on Automatic Control, Vol. 31, No. 7, 1986. pp. 869-870. doi:10.1109/TAC.1986.1104420

[12] T. Kaczorek, "Extensions of the Cayley-Hamilton Theorem for 2D Continuous-Discrete Linear Systems," Applied Mathematics and Computation Sciences, Vol. 4, No. 4, 1994, pp. 507-515.

[13] N. M. Smart and S. Brunett, "The Algebra of Matrices in n-Dimensional Systems," IMA Journal of Mathematical Control and Information, Vol. 6, No. 2, 1989, pp. 121-133. doi:10.1093/imamci/6.2.121

[14] M. Buslowicz and T. Kaczorek, "Reachability and Minimum Energy Control of Positive Linear Discrete-Time Systems with One Delay," Proceedings of $12^{\text {th }}$ Mediterranean Conference on Control and Automation, KasadesiIzmur, CD ROM, 2004.

[15] T. Kaczorek, "Linear Control Systems, Vol. I and II," Research Studies Press, Taunton, 1992-1993.

[16] B. G. Mcrtizios and M. A. Christodolous, "On the Generalized Cayley-Hamilton Theorem," IEEE Transactions on Automatic Control, Vol. 31, No. 1, 1986, pp. 156-157. 
[17] N. J. Theodoru, "M-Dimensional Cayley-Hamilton Theorem," IEEE Transactions on Automatic Control, Vol. 34, No. 5, 1989, pp. 563-565. doi:10.1109/9.24217

[18] M. Buslowicz, “An Algorithm of Determination of the Quasi-Polynomial of Multivariate Time-Invariant Linear System with Delays Based on State Equations," Archive of Automatics and Telemechanics, Vol. 36, No. 1, 1981, pp. 125-131.

[19] M. Buslowicz, "Inversion of Characteristic Matrix of the
Time-Delay Systems of Neural Type," Control Engineering, Vol. 7, No. 4, 1982, pp. 195-210.

[20] T. Kaczorek, "Extension of the Cayley-Hamilton Theorem for Continuous-Time Systems with Delays," International Journal of Applied Mathematics and Computer Science, Vol. 15, No. 2, 2005, pp. 231-234.

[21] T. Kaczorek, "Vectors and Matrices in Automation and Electrotechnics," Polish Scientific Publishers, Warsaw, 1988. 